\title{
An approach of implementing Application-as-a-Service (AaaS) model with Open-Source Applications
}

\author{
Article by ${ }^{1}$ Shahed Murshed and ${ }^{2}$ Mobarak Hossain \\ ${ }^{1}$ Information Technology, Texila American University, Bangladesh \\ ${ }^{2}$ Mobarak Hossain, Plexus Software \\ Email: s.murshed@texilaconnect.com
}

\begin{abstract}
Application as a Service (AaaS) is a service component of Cloud computing that falls in the category Software as a Service (SaaS). This paper presents an Application as a Service (AaaS) model using Open Source applications for File Sync, Share and Email solution for organizations. The proposed model enables the organizations to manage all computing resources involved totally by the service provider or by the company itself and the applications are facilitated as service on demand. The target group of the model developed is small organizations running in private cloud environment. We analyzed the common requirements of small scale organizations and that triggered us to develop an AaaS Service model with Open Source applications. This approach enables the organizations easy migration with minimal effort and with least cost.
\end{abstract}

Keywords: Application as a Service (AaaS), Open-Source Application, Application Services

\section{Introduction}

Applications as a Service (AaaS) is a cloud service where the model ensures delivery of computer software applications as a service to its users online i.e. via the Internet or Intranet. This type of software falls under one of the main core service - Software as a Service (SaaS). This approach of providing software as a service to the users is also known in the market as on-demand software. AaaS or on-demand software is becoming strong and a prominent entity in the software market due to its economical offering and efficiency that it can offer to organizations - big or small regardless. The approach of providing single software to multiple users confirms financial benefits to organizations by getting rid of the expenditure of individual user licenses and in the same time cutting the procurement, setup and operating cost of the hardware associated. As in AaaS model a software can be provided to multiple users simultaneously from a single point the efficiency is ensured both in the provider end and in the user end. This distribution is done through the Internet. There are different types of functions involved that are provided to the user and few to mention are: management of user accounts, provisioning of users, user subscription management and life cycle management of application. All these functions collectively make AaaS model a highly efficient and financially economical way to deliver software to small scale enterprise users. This approach is also effective for large organization within a private could environment.

Many Organizations use cloud based services to share valuable and confidential data within different departments of the organization and also in the same time with different entities outside of the organization. This type of services falls under the AaaS Cloud service and generally this services are rendered to the user by Companies that provide applications as a service (on-demand software). This type of provider are known as ASP or application service provider. ASPs own the software that they deliver to users and the users are billed based on use of the service. Even though this services are cost effective still for many organizations it is not possible to effort the services provided by the ASP due to financial shortcomings. ASPs bill on a per use basis during a month or can be billed in yearly basis and the approach thus makes software on demand. Our effort in this article is to present an AaaS Solution with Open Source applications, so that there is no charges involved for using the 
South American Journal of Academic Research

Special Edition May 2016

applications. Many organizations that wants to avail AaaS cloud services with no monthly cost involvement can adopt our solution. An important feature of this solution is that it is developed completely with Open Source applications and so only cost involved is for a small number of low cost hardware. For companies who are already running computer network can use the existing hardware in most cases which implies hardly any new expense to migrate to the proposed cloud services. Obviously being an Open Source application solution it is not that all services will be available in the solution but it will provide most commonly used applications by an organization. Our proposed model covers the following service applications: Office, Multimedia, Imaging, Email, Calendar, Contacts and File Manager. Apart from all those mentioned it offers few other services - Activity Log, Bookmarks and Data Usage Summery. The solution has been developed and the beta version has been running on test basis for Quantum Foundation of Bangladesh and is discussed later in the article.

\section{Types of Application Service Providers (ASPs)}

ASPs are divided into 4 major categories and is discussed in brief as stated below:

Specialist or Functional ASPs - this type of ASP is the one who provide a single application (e.g. solution for payroll of a company) to the client.

Vertical Market ASPs - provides a specific sets of software applications for a specific type of industry (e.g. application services for food service industry)

Enterprise ASPs - who provide customized applications for management of multiple functions within a single organization.

Local ASPs - who provide a variety of applications to small businesses in specific small geographical area.

\section{Where does AaaS stands in Cloud Computing}

Cloud computing is viewed as one of the most promising technologies in computing today. In the recent few years there have been significant development achieved in the IT industry especially in the field of virtualization techniques which triggered to more reliable services in cloud computing. Cloud computing concept and virtualization techniques together ensured cloud based services more affordable and as a result many organizations are leaning to this cloud service. Cloud computing solutions include IT infrastructure, development platforms and software.

IT Infrastructure (IaaS): The infrastructure of a cloud network is known as Infrastructure as a Service (IaaS). This service provides with the capability to provision processing, storage, networks, and other fundamental computing resources and with all these resources the user is able to deploy and run arbitrary software, which can include operating systems and applications.

Development Platform: This service is known as Platform as a Service (PaaS). PaaS is a cloud computing model that provides the user with software and applications over the Internet. In this model a Cloud Service Provider (CSP) delivers hardware and software tools as a service to the users particularly for application development. All the resources are hosted in the provider's side and as a result PaaS ensures no in-house hardware and software to develop.

Software: This service is known as Software as a Service (SaaS). SaaS is a software delivery method that provides access to software and its functions from any location through the Internet/Intranet. This service gives the user of the luxury of using expensive software with a fraction cost as SaaS pricing is based on a monthly fee with the fact that fact that the uses do not need any hardware as the software is hosted remotely in the providers end. From this point of view SaaS is also considered as Hosted Applications. The hosted applications are arranged by the ASPs to provide services to the clients as Application as a Service (AaaS). 


\section{Proposed AaaS Architecture}

As part of our AaaS architecture, we defined a model that shows how the system behaves from a user-centered design perspective. Our model is as shown in figure 1 below. AaaS for a particular application and for any integration work needed to deliver the service within this frame. The SaaS specialist is the technical resource who delivers any personalization, programming, and customization for it.

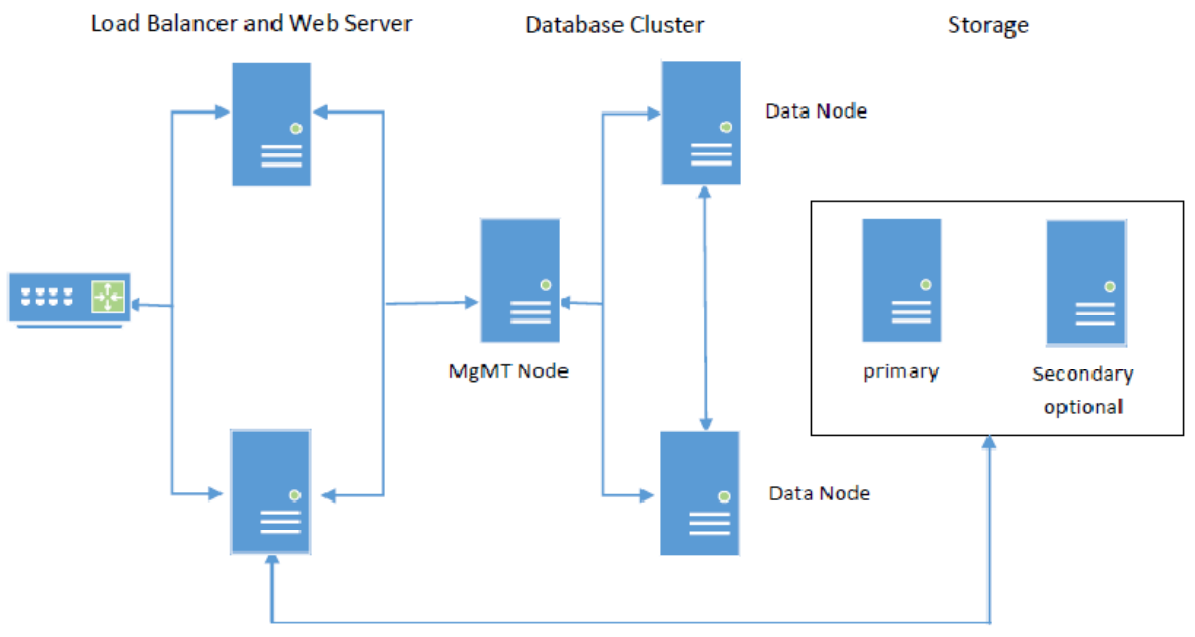

Figure 1: Cloud based Network Architecture for providing Application Service

In the model developed when a file share request is received the router sent the request to the load balancer and the load balancer forward it to the Management Node. After the request is further processed it is directed to the Data Node for appropriate interpretation in the database. Based on the request data is processed for retrieval to the user request point or in if required it is stored in the storage.

\section{Conceptual Architecture}

The conceptual architecture in figure 2 explains all the key capabilities required in the architecture designed, the logical separation of capabilities into tiers, and the logical set of services with varying capabilities. We do not expect that individual SaaS applications will necessarily include every service described in the conceptual architecture.

In the environment all the features are controlled by the IT Administrator who controls the whole environment. The attributes may by different based on the type of organization and depending on the nature of operation of the organization.

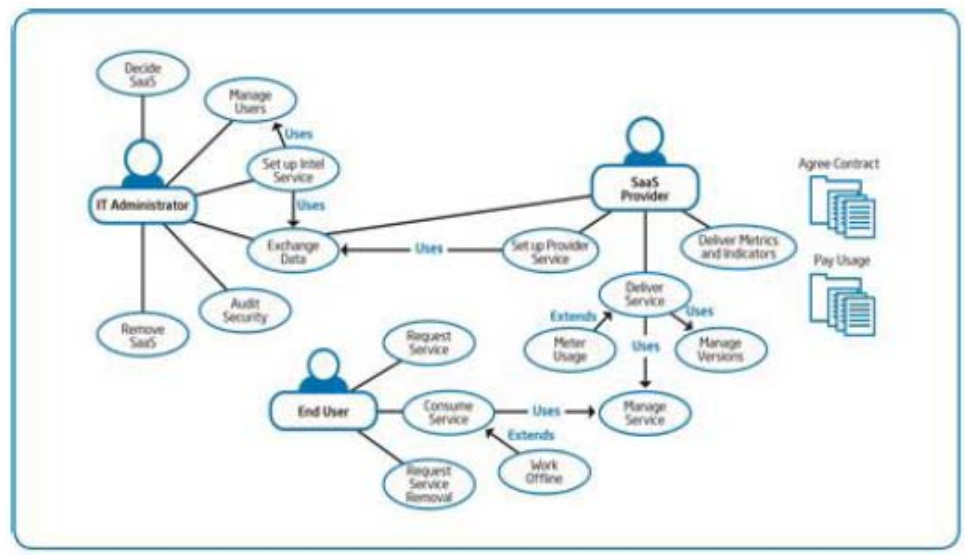

Figure 2. Software as a service (SaaS) architecture use-case model 
South American Journal of Academic Research

Special Edition May 2016

Therefore, the administrator needs to set the types of attributes necessary to run various operations involved. Once the administrator sets the policy as per requirement it is provided by the SaaS provider and once data is processed it is made available to the End User. Security is a major concern in any cloud environment. This is ensured by the IT Administrator assessing the risk factors based in the environment and using various IT Policies.

\section{Proposed Applications in the AaaS model}

Office Application Service: Word processing and Presentation, graphics and databases are the applications provided in this category. All the applications in this category can open and edit documents. In our model for word processing Documents 0.9.1 application service is considered.

Multimedia Service: Multimedia means representation of digital information in the form of audio, video, and animation. Information can be represented, stored, transmitted and processed digitally. When it comes to the term multimedia application it implies a collection of different types of text, graphics, images, audio and video. In our model for video processing we used videoJS subline 0.9.5 application and audio processing we used mp3 player 1.2.5 application.

Image Service: Image service allows display and analysis of image files. Most common features of this service includes querying, processing, previewing, downloading and adding. All those tasks mentioned are controlled through a set of image service parameter settings. For all image processing in our solution it is handled by gallery $+\mathbf{1 4 . 3 . 0}$ application.

Email Service: Email service is yet another essential service. This service provides webmail service to the user on-the-fly in any mobile device anywhere through Internet. Multiple accounts can be used in parallel and all the mailboxes are protected appropriate security features. Depending on the solution both POP and IMAP feature can be supported in the service provided. In our model for email read, compose, send and use other email related options we used mail template app. Email service is ensured using postfix 2.10.0 application.

Calendar Service: Calendar service is a very important application. It is a fast, effective tool for managing schedules. It keeps track of the upcoming appointments and keep the user on the track. Different calendar applications have different types of features like alarm for events, travel information, flight schedule, meetings, task management, schedule deliverables are some to mention. Calendar apps are becoming smarter and more functional. In our model for calendar service we used calendar app. calendar 0.6.6 application is used in our model to facilitate all the calendar features.

Contact Service: Contact service is the one that manages contacts and keep us connected with people. Contact app enables user to sort contact category wise, query contact, add and edit contacts, create group contacts, speed dial option from contact list, add/edit contacts with pictures etc. In our model for contact service we used contact app. In the model developed Contacts 0.5.0.0 application is used to manage all contact related features.

Activity: This service provides update and information of all activities associated with a user's files, such as sharing activity, updated, renamed, deleted and removed files. In our model for activity service we used activity 3.4.5 application.

Bookmarks: Bookmark service is an application that provides the user with the option of saving a webpage address. Frequently browsed web addresses can be saved and that expedite the browsing service more effective as data retrieval from some specific website is accessible faster. In our model for bookmarks app we used bookmarks 0.02.45 application.

Usage: Data usage monitoring is curtail for some organizations. Storage space is expensive and this is the usage service which up to date information on space being used by the user and that enable the user to manage files as per limit provided. In our model for usage check we used storage chart 3.4.5 application. 


\section{Case Study (Quantum Foundation of Bangladesh)}

Quantum Foundation of Bangladesh is a non-profitable organization that provides various types of training to its members. At the moment it has approximately 100000 members. They used to share ideas on training methods and materials through regular email and by meetings. We realized that if the solution we developed is implemented for them it could make the operation of the organization much easier. In this norm we proposed them to allow us to implement the system for them. They had been using server based network for file sharing. They had been using personal computer as well though internet and intranet. So, we used their existing infrastructure and implemented the design developed using the open source applications that we discussed in previous section. The applications that they are using at this time are office services, multimedia, imaging, email, calendar, contacts and activities. The applications are accessible from various client devices, through a thin client interface, such as a web browser (e.g. web-based e-mail). The members does not manage or control the underlying cloud infrastructure which includes server frames, work stations, software, storage, routers/switches and as well individual user specific application capabilities and configuration settings. The portal developed can be found at www.cloud.qmail.com.bd

\section{Conclusion}

The organizations that is adopting AaaS solution must consider the flexibility and risk management factors in reflection to their portfolios of IT services and the IT Infrastructure. AaaS clients need to consider the quality of the account monitoring tools a cloud AaaS provider makes available for the services it delivers. Even though the AaaS model is developed with a set of Open Source applications the user still should have the ability to determine when they have exceed their monthly usage limit for their subscription level. Clients should also have the ability to monitor the usage of individual users on an hourly basis and depending on daily basis so that data sync operation is not interrupted in time of need. The model developed indicates lack of administrative tools which may lead to data scare situation. Therefore, an effective administrative monitoring mechanism needs to be developed in the next level development. Another point that needs more attention is the authentication screen, even though it is ensured in the current version the templates needs to be improved which will be open for the next development. Many organizations use cloud-based services to share sensitive company data within the group members and in some cases with different vendors, partners and also at time with. They sync data with their workstations, personal devices and home computers, all in an effort to manage data with ease and with an efficient process - all without any intervention of IT's oversight. With the system developed one can effectively manage and protect data sets involved by hosting own solution on site, using own IT Infrastructure. This may not be suitable to hybrid could scenario but definitely a robust and efficient AaaS model for private cloud scenario.

\section{References}

[1.] Alberto Heredia, Ricardo Colomo-Palacios, Antonio de Amescua, "Software Business Models from a distribution perspective: A Systematic Mapping Study”, Conference on ENTERprise Information Systems / International Conference on Project MANagement / Conference on Health and Social Care Information Systems and Technologies, CENTERIS / ProjMAN / HCist 2015 October 7-9, 2015

[2.] Björn Johansson, Pedro Ruivo, Jorge Rodrigues, “Adoption Reasons for Enterprise Systems as a Service - A Recap of Provider Perspectives”, Conference on ENTERprise Information Systems / International Conference on Project MANagement / Conference on Health and Social Care Information Systems and Technologies, CENTERIS / ProjMAN / HCist 2015 October 7-9, 2015

[3.] Chou, W 2008, “Web Services: Software-as-a-Service (SaaS) Communicaton and Beyond”, IEEE Congress on Services.

[4.] E. Naone, Technology overview, conjuring clouds, MIT Technology Review, July-August, 2009.

[5.] http://apprenda.com/library/software-on-demand/applications-as-a-service/ 
South American Journal of Academic Research Special Edition May 2016

[6.] https://azure.microsoft.com/en-us/documentation/articles/choose-web-site-cloud-service-vm/ [7.] https://devcentral.f5.com/articles/f5-synthesis-delivering-web-application-security-in-the-cloud-asa-service

[8.] http://www.intel.com/content/www/us/en/cloud-computing/cloud-computing-intel-it-architectingsoftware-as-a-service-paper.html

[9.] https://owncloud.com/owncloud-architecture-overview/

[10.] Mobarak Hossain holds B.Sc. in Software Engineering, He was a scientist trainee at Daimler Chrysler in Bangalore, India. He has been involved in research projects in Cloud Computing in the area of OpenStack Cloud Platform and Cloud Virtualization Techniques.

[11.] National Institute of Standards and Technology, The NIST Definition of Cloud Computing, Information Technology Laboratory, 2009.

[12.] Kulkarni, G, Gambhir, J \&Palwe, R 2012, “Cloud Computing-Software As Service”, International Journal of Cloud Computing And Services Science, Vol. 1, No. 1, pp.11-16.

[13.] Rajkumar, B. Yeo, C. Venugopal, S. Malpani, S. Cloud computing and emerging IT platforms: vision, hype, and reality for delivering computing as the 5thutility, Future Generation Computer Systems (2009).

[14.] Reese, G. Cloud Application Architectures: Building Applications and Infrastructure in the Cloud, in: Theory in Practice, O’Reilly Media, 2009.

[15.] Ruivo, P., V. Santos, and T. Oliveira, Data protection in services and support roles - a qualitative research amongst ICT professionals. Procedia Technology 2014. 9: p. 94-99.

[16.] Satyanarayana, S 2012, “CLOUD COMPUTING: SAAS”, Journal of Computer Science and Telecommunications, Vol. 4, No. 4, pp. 76-79.

[17.] Seethamraju, R., Adoption of Software as a Service (SaaS) Enterprise Resource Planning (ERP) Systems in Small and Medium Sized Enterprises (SMEs). Information Systems Frontiers, 2014. 8: p. 118.

[18.] Shahed Murshed holds a B.Sc. in Computer Science (Computer Engineering), an M.Eng in Internetworking and is currently pursuing Ph.D. in Computer Science at the Texila American University, School of Information Technology. He has been involved in research projects in Cloud Computing in the area of OpenStack Cloud Platform, IaaS Network Infrastructure development and virtualization in Cloud Computing.

[19.] Stanoevska-Slabeva, K. Wozniak, T. Grid and Cloud Computing-A Business Perspective on Technology and Applications, Springer-Verlag, Berlin, Heidelberg, 2010. 